ELECTRONIC RESEARCH ANNOUNCEMENTS OF THE AMERICAN MATHEMATICAL SOCIETY

Volume 3, Pages 52-54 (July 29, 1997)

S $1079-6762(97) 00023-1$

\title{
CLASSIFICATION OF COMPACT HOMOGENEOUS SPACES WITH INVARIANT SYMPLECTIC STRUCTURES
}

\author{
DANIEL GUAN
}

(Communicated by Gregory Margulis)

\begin{abstract}
We solve a longstanding problem of classification of compact homogeneous spaces with invariant symplectic structures. We also give a splitting conjecture on compact homogeneous spaces with symplectic structures (which are not necessarily invariant under the group action) that makes the classification of this kind of manifolds possible.
\end{abstract}

A smooth manifold $M$ equipped with a smooth transitive action of a Lie group is called a homogeneous space. If in addition $M$ is a symplectic manifold, we refer to it as a homogeneous space with a symplectic structure and, if the structure is invariant, a homogeneous space with an invariant symplectic structure.

Recently there has been much progress in the area of symplectic manifolds and group actions. However, the classical problem of classifying compact homogeneous spaces with symplectic structure is seemingly unreachable.

The paper [DG] did give us some hope in this direction and [Gu1], [Gu2], [Hk] provide a nice picture for compact complex homogeneous spaces with a symplectic structure. The following result can be found in $[\mathrm{DG}]$ (see also $[\mathrm{Hk}]$ for a "simpler" proof):

Proposition 1 ([DG], [Hk]). Every compact homogeneous pseudo-Kähler manifold is a product of a torus and a rational homogeneous space, both with standard pseudoKähler structures.

We note here that the proof of this result is more complicated than that of the Kähler case in [Mt], since in the Kähler case, by the compactness of the manifold, one can easily see that a compact Lie group acts transitively and the isotropy group is a subgroup of an orthogonal group, i.e., both groups are reductive.

In [Gu1], [Gu2] we observed that the method in [Hk] actually works for a compact complex homogeneous space with an invariant symplectic structure:

Received by the editors February 21, 1997.

1991 Mathematics Subject Classification. Primary 53C15, 57S25, 53C30; Secondary 22E99, $15 \mathrm{~A} 75$.

Key words and phrases. Invariant structure, homogeneous space, product, fiber bundles, symplectic manifolds, splittings, prealgebraic group, decompositions, modification, Lie group, symplectic algebra, compact manifolds, uniform discrete subgroups, classifications, locally flat parallelizable manifolds.

Supported by NSF Grant DMS-9401755 and DMS-9627434.

(C) 1997 American Mathematical Society 
Proposition 2. Every compact complex homogeneous space with an invariant symplectic structure is a product of a torus and a rational homogeneous space, both with standard symplectic structures.

And we also proved the following theorem:

Proposition 3. Every compact homogeneous complex manifold with a 2-cohomology class $\omega$ such that $\omega^{n}$ is not zero in the top cohomology is a product of a rational homogeneous space and a complex parallelizable solv-manifold with a right invariant symplectic structure on its universal covering.

This generalized the result of [BR] for the Kähler case (one does not assume that the Kähler form is invariant).

These results suggest further study in two directions along the lines of Proposition 3. One is the classification of compact complex homogeneous spaces; the other is the classification of compact homogeneous spaces with a symplectic structure. The first problem can be solved by the method in [Gu3], where we prove that every compact complex homogeneous space with an invariant volume is a torus fiber bundle over a product of a rational homogeneous space and a complex parallelizable manifold. In the present paper we announce our result in the direction of the second problem. This is quite analogous to the results in [Gu1], [Gu2]:

Main Theorem. Every compact homogeneous space with an invariant symplectic structure is a product of a rational homogeneous space and a torus with invariant symplectic structures.

In this classification, the tori which occur are not necessarily standard. One might use such a nonstandard torus to obtain new symplectic manifolds as in [Bo] and [Gu1]. The following conjecture arises naturally from our arguments for the proof of the above theorem:

Conjecture. If $G / H$ is a compact homogeneous space with a symplectic structure, then $G / H$ is a product of a rational homogeneous space and a compact locally flat parallelizable manifold with a symplectic structure.

Here we call a manifold $N$ locally flat parallelizable if $N=G / H$ for a simply connected Lie group $G$ which is diffeomorphic to $\mathbf{R}^{k}$ for some integer $k$ and $H$ is a uniform discrete subgroup.

In our future work we will attempt to prove this conjecture and to classify the compact locally flat parallelizable manifolds with this additional structure.

\section{ACKNOWLEDGEMENT}

We thank the referee for his gracious advice on the final exposition of this paper.

\section{REFERENCES}

[Bo] F. A. Bogomolov, On Guan's examples of simply connected non-Kähler compact complex manifolds, Amer. J. Math. 118 (1996), 1037-1046. CMP 97:01

[BR] A. Borel and R. Remmert, Über kompakte homogene Kählersche Mannigfaltigkeiten, Math. Ann. 145 (1962), 429-439. MR 26:3088

[DG] J. Dorfmeister and Z. Guan, Classifications of compact homogeneous pseudo-Kähler manifolds, Comm. Math. Helv. 67 (1992), 499-513. MR 93i:32042

[Gu1] Z. Guan, Examples of compact holomorphic symplectic manifolds which admit no Kähler structure. In Geometry and Analysis on Complex Manifolds-Festschrift for Professor S. Kobayashi's 60th Birthday, World Scientific, 1994, pp. 63-74. 
[Gu2] D. Guan, A splitting theorem for compact complex homogeneous spaces with a symplectic structure, Geom. Dedi. 67 (1996), 217-225. CMP 97:02

[Gu3] D. Guan, Classification of compact complex homogeneous spaces with invariant volumes, preprint 1996.

[Gu4] D. Guan, Examples of compact holomorphic symplectic manifolds which are not Kählerian II, Invent. Math. 121 (1995), 135-145. CMP 95:16

[Hk] A. T. Huckleberry, Homogeneous pseudo-Kählerian manifolds: a Hamiltonian viewpoint, preprint, 1990.

$[\mathrm{Kb}] \quad \mathrm{S}$. Kobayashi, Differential geometry of complex vector bundles, Iwanami Shoten Publishers and Princeton University Press, 1987. MR 89e:53100

[Mt] Y. Matsushima, Sur les espaces homogènes kählériens d'un groupe de Lie réductif, Nagoya Math. J. 11 (1957), 53-60. MR 19:315c

[Ti] J. Tits, Espaces homogènes complexes compacts, Comm. Math. Helv. 37(1962), 111-120. MR 27:4248

Department of Mathematics, Princeton University, Princeton, NJ 08544

E-mail address: zguan@math.princeton.edu 